Tecno Lógicas

ISSN 0123-7799

Vol. 17, No. 32, pp. 21-31

Enero-junio de 2014
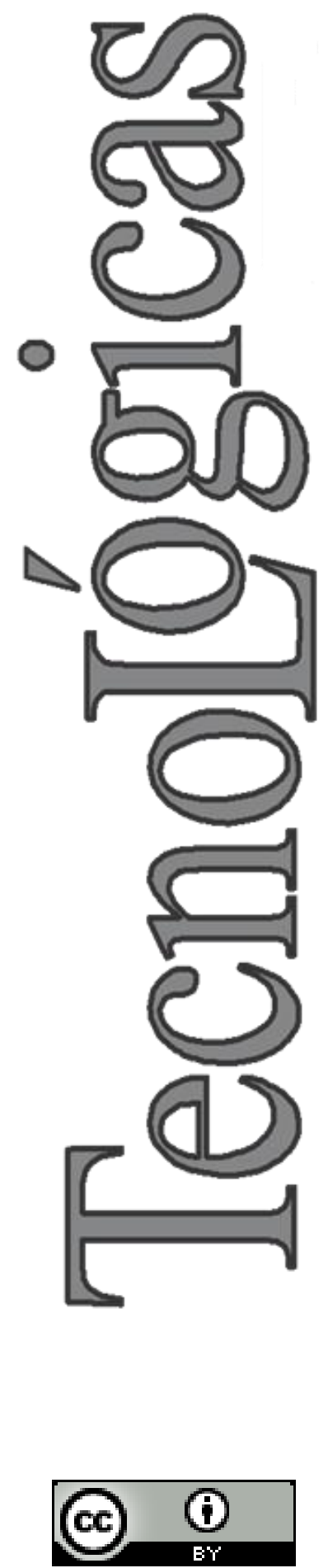

\section{Efecto de las políticas de comercio y regulación en el nivel de satisfacción de los usuarios de telefonía móvil en Colombia}

\section{Effect of trade and regulatory policies on level users satisfaction of mobile phone service in Colombia}

\author{
Karen M. Prada-Ávila¹, Silvia I. Díaz-Rueda², \\ Néstor A. Nova-Arévalo 3
}

Recibido: 22 de agosto de 2013, Aceptado: 22 de noviembre de 2013

Como citar / How to cite

K. M. Prada-Ávila, S. I. Díaz-Rueda y N. A. Nova-Arévalo, "Efecto de las políticas de comercio y regulación en el nivel de satisfacción de los usuarios de telefonía móvil en Colombia”, Tecno Lógicas, vol. 17, no. 32, pp. 21-31, 2014.

1 Ingeniera Industrial, Ingeniería industrial, Facultad de Ingeniería, Universidad Cooperativa de Colombia - Sede Bogotá, BogotáColombia, karen.pradaa@campusucc.edu.co

2 Ingeniera Industrial, Ingeniería industrial, Facultad de Ingeniería, Universidad Cooperativa de Colombia - Sede Bogotá, Bogotá-

Colombia, silvia.diazr@campusucc.edu.co

3 M.Sc. en Ingeniería Industrial, Ingeniería industrial, Facultad de Ingeniería, Universidad Cooperativa de Colombia - Sede Bogotá, Bogotá-Colombia, nestor.nova@campusucc.edu.co 
K. M. Prada-Ávila et al. / Efecto de las políticas de comercio y regulación en el nivel de satisfacción de los usuarios de telefonía móvil en Colombia

\section{Resumen}

En esta investigación se identificó y cuantificó el efecto de las políticas legislativas, normativas y de comercio en el nivel de satisfacción de los usuarios del servicio de telefonía móvil en Colombia. Para ello se realizó un análisis de mercado y comercio de la telefonía móvil, así como de las políticas legislativas, normativas y de calidad y satisfacción de los usuarios del servicio en Colombia, para el periodo 2000-2012. Como resultado se formularon siete modelos matemáticos del nivel de satisfacción en el país y del segmento prepago y postpago de los tres operadores con mayor participación en el mercado, a través de tres factores como son las cifras de importaciones de teléfonos móviles, la cantidad de teléfonos homologados y la distribución del mercado entre operadores, y se estimaron los niveles no reportados por el regulador para el periodo 2010-2012, encontrando una tendencia constante cercana al 70\%. Se concluye que en el mercado de la telefonía móvil existen efectos regulatorios adicionales a los medidos y declarados por regulador, los cuales limitan la garantía de un nivel mínimo de calidad del servicio como requisito de operación dentro del mercado. La presencia del factor de importación en todos los modelos refleja los efectos de la evolución del mercado sobre la satisfacción de los usuarios del servicio de telefonía móvil, dados por la implementación de nuevas tecnologías, la oferta de nuevos servicios y la participación de nuevos operadores.

\section{Palabras clave}

Satisfacción de usuarios, calidad del servicio, regulación, telefonía móvil celular, operadores de telefonía.

\section{Abstract}

This research characterized and quantified the effect of the legislative policy, regulations, and trade policies in the level users satisfaction of the mobile phone service in Colombia. For this, we conducted a market and commerce analysis of mobile telephone, as well as legislative, regulatory, quality and satisfaction policies of service users in Colombia, for the period 2000-2012. As a result seven mathematical models were formulated of the level users satisfaction in Colombia and prepaid and postpaid segment of the three operators with greater market share, through three factors like value of importation of mobile phones, quantity of phones approved and market sharing between operators, levels not reported by regulator were estimated for the period 2010-2012 and a consistent trend of about $70 \%$ was discovered. We conclude that in the mobile phone market there are regulatory effects additional to the measured and reported by the regulator, which limit the guarantee of a minimum level of quality of service as an operational requirement within the market. The presence of the importation factor in all models reflects the effects of market evolution on the user satisfaction of mobile phone service, given by the implementation of new technologies, new services offers and the participation of new mobile phone operators.

\section{Keywords}

User satisfaction, quality of service, regulation, mobile phone, mobile phone operators. 
K. M. Prada-Ávila et al. / Efecto de las políticas de comercio y regulación en el nivel de satisfacción de los usuarios de telefonía móvil en Colombia

\section{INTRODUCCIÓN}

El presente documento tiene como objetivo central hacer un análisis al mercado de la telefonía móvil en Colombia para identificar el efecto que tienen las políticas legislativas, normativas $\mathrm{y}$ de comercio sobre el Nivel de Satisfacción de los Usuarios - NSU.

En este estudio cobra especial relevancia la gestión de las diferentes entidades del Estado como la DIAN, DANE, MINTIC entre otras para garantizar el buen funcionamiento del servicio en las telecomunicaciones dentro del país, además del marco legal y regulatorio. Por lo anterior, en este estudio se determinaron aspectos complementarios de la calidad del servicio que pueden ser tenidos en cuenta dentro de las políticas normativas que rigen el funcionamiento y servicio de las telecomunicaciones. En la investigación se tuvieron en cuenta variables económicas, de calidad y comercio que afectan la calidad del servicio de telefonía móvil en el país. Con las variables identificadas se formularon siete modelos estadísticos de regresión que permiten determinar la magnitud del efecto que éstas causan en el nivel de satisfacción de los usuarios.

Esta investigación da respuesta a la pregunta ¿cuál es el efecto de las políticas legislativas, normativas y de comercio del servicio de telefonía móvil sobre el nivel de satisfacción de los usuarios en Colombia?, por lo tanto el objetivo principal consistió en analizar el comportamiento del mercado de la telefonía móvil en Colombia para identificar y cuantificar el efecto que tienen las políticas legislativas, normativas y de comercio de telecomunicaciones móviles en Colombia sobre el Nivel de Satisfacción de los Usuarios, a través de técnicas de análisis estadístico inferencial.

Esto se logró por medio de tres objetivos específicos: primero la determinación de los requerimientos generales de importación y certificación de teléfonos móviles, segundo el análisis de los mecanismos de medición del NSU en Colombia y en otros países y el modelamiento y análisis de la relación entre los resultados del primero y segundo objetivo.

En el marco de referencia conceptual utilizado en esta investigación y presentado en Nova y Pinzón [1] y Nova et al. [2], fueron integradas tres áreas del conocimiento como son: la economía, la psicología y la cibernética para formular modelos matemáticos que identifican, explican $\mathrm{y}$ cuantifican el efecto regulador sobre la racionalidad económica de los usuarios del servicio de telefonía móvil celular en Colombia.

Así mismo, otro referente para esa investigación se encuentra en Nova et al. [3], en donde se analizó estadísticamente el efecto del regulador global y local de la telefonía móvil celular en el comportamiento económico del mercado de varios países y regiones en el mundo, esto se hace calculando las cuasi rentas recibidas por los operadores del servicio en función de su participación en el mercado. De esta manera se distingue el efecto global de los reguladores y se define un indicador de elección y toma de decisiones para las instituciones reguladoras, operadores y para los usuarios del servicio.

\subsection{Los operadores del servicio en Colombia}

El estado colombiano en observancia de los artículos 75, 101, 102 de la Constitución Política de 1991 y en cumplimiento de la ley 37 de 1993 concesionó el servicio de la telefonía móvil celular para la prestación de este servicio en el territorio colombiano. Por su parte el Decreto 990/1998 en su capítulo IV habla sobre la naturaleza del contrato de servicios y establece que las relaciones entre los suscriptores y los operadores se regirán por lo dispuesto en el contrato que celebren para la prestación del servicio de Telefonía Móvil Celular.

Los contratos de concesión vigentes para prestar el servicio de telefonía móvil celular fueron suscritos hasta el 2014 con 
K. M. Prada-Ávila et al. / Efecto de las políticas de comercio y regulación en el nivel de satisfacción de los usuarios de telefonía móvil en Colombia

Telefónica móviles con su marca Movistar y América móvil con su marca Comcel (Claro) y el suscrito con Colombia Móvil con su marca Tigo hasta el 2013.

\begin{tabular}{cccc}
\multicolumn{4}{c}{ Tabla 1. Ingresos de los operadores móviles (millones de } \\
pesos). Fuente: [4] \\
\hline Año & CLARO & MOVISTAR & TIGO \\
\hline 2000 & $\$ 409.532$ & $\$ 416.965$ & \\
2001 & $\$ 576.136$ & $\$ 496.163$ & \\
2002 & $\$ 868.877$ & $\$ 716.001$ & \\
2003 & $\$ 984.704$ & $\$ 941.846$ & \\
2004 & $\$ 1.074 .494$ & $\$ 1.112 .514$ & $\$ 328.068$ \\
2005 & $\$ 1.776 .954$ & $\$ 1.222 .019$ & $\$ 542.343$ \\
2006 & $\$ 2.758 .254$ & $\$ 1.240 .356$ & $\$ 591.144$ \\
2007 & $\$ 3.382 .418$ & $\$ 1.362 .645$ & $\$ 696.969$ \\
2008 & $\$ 3.586 .688$ & $\$ 1.564 .615$ & $\$ 650.895$ \\
2009 & $\$ 3.572 .596$ & $\$ 1.369 .348$ & $\$ 664.761$ \\
2010 & $\$ 3.765 .255$ & $\$ 1.548 .257$ & $\$ 690.789$ \\
\hline
\end{tabular}

La Tabla 1 muestra los ingresos anuales registrados por el Sistema de Información Unificado del Sector de Telecomunicaciones - SIUST de cada uno de los operadores móviles vigentes en Colombia, siendo el operador Claro el que posee mayores ingresos en el país. El índice de penetración en el mercado y la cantidad de líneas activas en cada operador para el periodo comprendido entre 2000 y 2012 se relacionan en la Tabla 2; en ésta se observa un aumento constante del nivel de consumo del servicio, registrando para el año 2012 un índice de penetración de $102,8 \%$ lo cual indica que existe más de una línea celular por habitante.

En función del índice de penetración del servicio en Colombia, la CRCom a través de la Resolución 1672 del 2006 y la Circular 060 del 2007 estableció como requisito previo a la venta de dichos equipos, la presentación de un certificado de conformidad de norma técnica de conexión a la red (GSM y CMDA: FCC parte 22, subparte H banda $850 \mathrm{MHz}$; FCC parte 24 banda 1,9 GHz y AMPS/TMDA: Estándares EIA/TIA aplicables y FCC parte 22, subparte $\mathrm{H}$ y norma técnica de radiación (IEEE Std C95.1 o ICNIRP, conforme a la recomendación UIT-T K.52) expedido por aquellos organismos (laboratorios) que se encuentran reconocidos a nivel nacional o internacional.

Por otra parte, la distribución del mercado refleja la posición dominante de Claro durante todo el periodo, lo cual es objeto de análisis dada la contradicción legal y económica entre la libre competencia que proclaman las leyes colombianas y el monopolio consentido por la regulación estatal. Las líneas activas son el número líneas prepago y postpago que registran actividad monetaria para los operadores.

Tabla 2. Distribución del mercado de telefonía en Colombia. Fuente: [4]

\begin{tabular}{cccccc}
\hline Año & Índice de penetración & CLARO & MOVISTAR & TIGO & Líneas activas \\
\hline 2000 & - & - & - & - & 2.049 .268 \\
2001 & - & - & - & - & 3.265 .261 \\
2002 & $0,4 \%$ & - & - & - & 4.596 .594 \\
2003 & $0,3 \%$ & $59,4 \%$ & $33,7 \%$ & $6,9 \%$ & 6.186 .206 \\
2004 & $0,4 \%$ & $55,7 \%$ & $31,6 \%$ & $12,7 \%$ & 10.400 .578 \\
2005 & $50,9 \%$ & $64,1 \%$ & $27,6 \%$ & $9,3 \%$ & 21.849 .993 \\
2006 & $63,8 \%$ & $64,2 \%$ & $28,0 \%$ & $7,8 \%$ & 29.762 .715 \\
2007 & $73,5 \%$ & $64,8 \%$ & $25,9 \%$ & $9,3 \%$ & 33.941 .118 \\
2008 & $91,7 \%$ & $66,0 \%$ & $24,0 \%$ & $9,7 \%$ & 41.364 .753 \\
2009 & $93,4 \%$ & $67,0 \%$ & $21,7 \%$ & $10,9 \%$ & 41.154 .630 \\
2010 & $97,7 \%$ & $66,1 \%$ & $22,5 \%$ & $11,0 \%$ & 44.477 .653 \\
2011 & $100,3 \%$ & $65,9 \%$ & $22,1 \%$ & $11,6 \%$ & 46.200 .421 \\
2012 & $102,8 \%$ & $65,0 \%$ & $21,7 \%$ & $12,8 \%$ & 47.172 .785 \\
\hline
\end{tabular}


K. M. Prada-Ávila et al. / Efecto de las políticas de comercio y regulación en el nivel de satisfacción de los usuarios de telefonía móvil en Colombia

\subsection{Nivel de Satisfacción de los Usuarios (NSU) de telefonía móvil}

Colombia ha avanzado con paso firme en el acelerado proceso de transformación de las telecomunicaciones. El servicio de telefonía celular (TMC) ha registrado los mayores niveles de crecimiento en cobertura y generación de ingresos tanto para la industria como para la nación como ningún otro servicio de telecomunicaciones.

La Constitución Política de Colombia y el actual marco legal conciben el deber de garantizar la adecuada prestación del servicio de telefonía por parte de las instituciones del estado. Entonces resulta útil determinar las debilidades tanto en las entidades públicas como en los operadores que pueden limitar la adecuada prestación del servicio y afectar el nivel de satisfacción en los usuarios (NSU), a fin de establecer acciones concretas por parte de los actores involucrados que conlleven a un mejoramiento en la prestación del servicio de telefonía móvil en el país.

$\mathrm{Al}$ hablar de Calidad en el ámbito de los servicios de telecomunicaciones se puede hacer una aproximación al tema desde el punto netamente técnico, como la calidad del funcionamiento de la red, o desde la calidad del servicio que percibe el usuario y que según, la Unión Internacional de Telecomunicaciones - UIT en [5], se define como: "El efecto colectivo del funcionamiento del servicio que determina el grado de satisfacción del usuario de un servicio". Desde la perspectiva del usuario, periódicamente se efectúa la medición del NSU de los servicios de telecomunicaciones, dicha medición es llevada a cabo por la Comisión de Regulación de las Comunicaciones CRCom, a través de firmas de consultoría.

Por principios de calidad en el servicio y equidad en una transacción comercial, se establece que los servicios de telefonía móvil celular se deben prestar de forma continua y eficiente, cumpliendo con las normas de calidad establecidas en el con- trato de concesión y las normas que regulan el servicio.

Sin embargo y desafortunadamente para los usuarios del servicio de telefonía móvil, en Colombia aún no existe una obligación regulatoria sobre un nivel de cumplimiento mínimo en el indicador del NSU para los operadores de dichos servicios.

\subsection{Método de medición del NSU en Colombia}

En Colombia, el NSU de telefonía móvil se mide a través del manual de medición elaborado por la Comisión de Regulación de las Comunicaciones - CRCom para los servicios de Telefonía Móvil Celular - TMC, Servicios de Comunicación Personal - PCS y servicios de acceso troncalizado Trunking tal como se muestra en [6].

El manual de medición se utiliza como herramienta de consulta en el desarrollo de la investigación específica sobre el NSU de dichos servicios para los segmentos prepago y postpago.

Este indicador del NSU se sustenta en una base conceptual según la cual el modelo de medición del nivel de satisfacción del usuario de un servicio o producto supone la existencia de un constructo hipotético llamado "Satisfacción del Usuario", el cual debe ser inferido a partir de la indagación sobre diferentes elementos de la experiencia de servicio que habitualmente vive el usuario (atributos de calidad o elementos de satisfacción) los cuales se enuncian como preguntas de un cuestionario ante las cuales el entrevistado expresa su calificación que le da de acuerdo con la calidad percibida en ellos como se evidencia en [6].

El modelo de satisfacción del usuario se compone de una ecuación lineal, como en (1), e incluye un conjunto de ponderadores para cada uno de los atributos (variables independientes), los cuales representan el impacto, importancia o peso relativo que tienen en la predicción de la experiencia de satisfacción general del usuario (variable 
K. M. Prada-Ávila et al. / Efecto de las políticas de comercio y regulación en el nivel de satisfacción de los usuarios de telefonía móvil en Colombia

dependiente), una explicación más amplia se encuentra en [6].

$N S U=\left(\beta_{1} A_{1}+\beta_{2} A_{2}+\beta_{3} A_{3}+\cdots+\beta_{n} A_{n}\right)$

Dónde $\left(\beta_{i}\right)$ es la importancia relativa o aporte del atributo i-esimo a la explicación de la satisfacción general del usuario, $\left(A_{i}\right)$ es el atributo i-esimo, $\mathrm{n}$ es el número total de variables independientes que tenga el modelo y NSU es el Nivel de Satisfacción del Usuario. Los atributos de calidad del servicio que se miden según el manual son: Calidad en la comunicación, atención al cliente, respuesta a solicitudes, consultas y reclamos, planes, promociones y equipos, facturación y pago, relación costo-beneficio.

El proceso de medición del NSU obedece a técnicas de muestreo por aleatorización, por lo tanto existirán tantos marcadores de NSU como usuarios hayan sido encuestados. Luego de calculado el NSU por usuario $\left(N S U_{k}\right)$ se obtiene el NSU del servicio $\left(N S U_{h}\right)$, como en $(2)$.

$N S U_{h}=\sum_{k=1}^{n} \frac{N S U_{k}}{n_{h}}$

Dónde $N S U_{h}$ es el nivel de satisfacción global del servicio h, $N S U_{k}$ es el nivel de satisfacción obtenido para cada usuario y $n_{h}$ es la cantidad total de usuarios encuestados del servicio h.

\subsection{Resultados de la medición del NSU en Colombia}

La Tabla 3 muestra los resultados de la medición del NSU a partir del año 2000 hasta el 2009 especificando el porcentaje de cada operador en el plan de postpago y prepago. Valores del NSU para años posteriores al 2009 no han sido reportados por la CRCom a la fecha de esta investigación y se desconoce si las medidas fueron realizadas.

El análisis de esta tabla indica una variación promedio de 3,8\% en el NSU global respecto al incremento promedio anual del $47,2 \%$ en el número de líneas activas. El periodo en el cual se presentó el mayor crecimiento del NSU se dio entre 2007 y 2006 con una variación positiva de 22,9 , mientras que un descenso de $13,7 \%$ se registró entre 2004 y 2005 el cual se contrapone al comportamiento en el número de líneas que para el mismo periodo se duplicó; del año 2007 al 2009 se registró un freno en el NSU con una pequeña variación de $0,4 \%$.

Tabla 3. NSU en el periodo 2000-2009. Fuente: [7]

\begin{tabular}{|c|c|c|c|c|c|c|c|c|}
\hline \multirow{2}{*}{ Año } & \multirow{2}{*}{ Líneas activas } & \multirow{2}{*}{ NSU Colombia } & \multicolumn{2}{|c|}{ NSU Claro } & \multicolumn{2}{|c|}{ NSU Movistar } & \multicolumn{2}{|c|}{ NSU Tigo } \\
\hline & & & Pre & Pos & Pre & Pos & Pre & Pos \\
\hline 2000 & 2.049 .268 & $55,0 \%$ & & & & & & \\
\hline 2001 & 3.265 .261 & $58,5 \%$ & $58,4 \%$ & $46,0 \%$ & $57,0 \%$ & $52,0 \%$ & & \\
\hline 2002 & 4.596 .594 & $56,2 \%$ & $51,7 \%$ & $43,0 \%$ & $55,7 \%$ & $54,0 \%$ & & \\
\hline 2003 & 6.186 .206 & $62,0 \%$ & $56,2 \%$ & $57,6 \%$ & $66,6 \%$ & $60,9 \%$ & $56,6 \%$ & $52,4 \%$ \\
\hline 2004 & 10.400 .578 & $65,6 \%$ & $62,9 \%$ & $57,6 \%$ & $66,6 \%$ & $60,8 \%$ & $56,6 \%$ & $52,4 \%$ \\
\hline 2005 & 21.849 .993 & $56,6 \%$ & $60,4 \%$ & $56,0 \%$ & $56,0 \%$ & $51,2 \%$ & $53,3 \%$ & $50,0 \%$ \\
\hline 2006 & 29.762 .715 & $59,8 \%$ & $63,6 \%$ & $60,4 \%$ & $55,4 \%$ & $55,6 \%$ & $61,2 \%$ & $58,9 \%$ \\
\hline 2007 & 33.941 .118 & $73,5 \%$ & $76,4 \%$ & $75,9 \%$ & $76,9 \%$ & $73,3 \%$ & $77,7 \%$ & $71,2 \%$ \\
\hline 2008 & 41.364 .753 & $73,6 \%$ & $78,2 \%$ & $73,3 \%$ & $76,7 \%$ & $73,6 \%$ & $73,8 \%$ & $72,8 \%$ \\
\hline 2009 & 41.154 .630 & $73,9 \%$ & $76,3 \%$ & $72,9 \%$ & $75,9 \%$ & $69,1 \%$ & $78,2 \%$ & $72,7 \%$ \\
\hline 2010 & 44.477 .653 & & & & & & & \\
\hline 2011 & 46.200 .421 & & & & & & & \\
\hline 2012 & 47.172 .785 & & & & & & & \\
\hline
\end{tabular}


K. M. Prada-Ávila et al. / Efecto de las políticas de comercio y regulación en el nivel de satisfacción de los usuarios de telefonía móvil en Colombia

Respecto a las mediciones particulares se puede mencionar que el NSU promedio de los usuarios del servicio prepago es del $65 \%$ mientras que el NSU postpago es del $60 \%$, esto indica que aquellos usuarios por demanda están ligeramente más satisfechos que aquellos que firman un contrato de servicio con los operadores, este comportamiento se replica para las mediciones de cada operador.

Para ampliar el panorama del lector, en el año 2009, mediciones del NSU en Chile mostraron un promedio de $71,8 \%$ para los tres operadores que agrupan la mayor cantidad del mercado como son Entel PCS, Movistar y Claro según el Centro Micro Datos en [8]. En España, país con una demanda de servicios similar a la Colombiana pero que triplica el número de operadores del servicio, la medición para el mismo año arrojo un NSU promedio de $70,18 \%$, como se observa en [9], para Movistar, Vodafone, Orange, Yoigo, y otras.

\section{METOdOLOGíA}

Esta investigación se desarrolló en dos fases, la primera siguió tres pasos como son: búsqueda bibliográfica sobre las disposiciones legales de importación, certificación y homologación de teléfonos móviles, luego se analizó el mercado de las telecomunicaciones móviles en Colombia identificando los índices de penetración de cada operador, el número de líneas activas, ingresos anuales, entre otras. Por último se analizó el método de medición del Nivel de Satisfacción de los Usuarios - NSU de telefonía móvil y se recolectaron los resultados de medición de éste para el periodo 20002012.

De esta fase se obtuvieron 39 variables de estudio agrupadas en las siguientes unidades de análisis con su respectiva codificación:

Siete variables dependientes: Total NSU - NSU total del país incluido segmen- tos prepago y postpago, NSU pre Com NSU prepago Claro, NSU pre Tel - NSU prepago Telefónica, NSU pre Tigo - NSU prepago Tigo, NSU post Com - NSU postpago Claro, NSU post Tel - NSU postpago Telefónica, NSU post Tigo - NSU postpago Tigo.

32 variables independientes: C1-Cifras de Importaciones según DIAN, C2-MINTIC Q Abonados, C3-MINTIC Q Abonados_C, C4-CRC Tel homologados, C5-CRC Tel homologados_C, C6-DANE Hogares con cel, P1-Penetración en el mercado Total, P2-Penetración en el mercado Claro, P3Penetración en el mercado Claro_C, P4Penetración en el mercado Movistar, P5Penetración en el mercado Tigo, P6Penetración en el mercado Uff, P7Penetración en el mercado otros, I1Cantidad Importaciones DIAN, I2-Valor de Importaciones DIAN en U\$, I3-Cantidad Importaciones DANE, I4-Valor de Importaciones DANE en US, I5-Valor de Importaciones CRC en US, I6-Cantidad Importaciones CRC, I7-Ventas de teléfonos según MINTIC, Ing1-Ingresos Claro, Ing2Ingresos Claro_C, Ing3-Ingresos Movistar, Ing4-Ingresos Tigo, Pqrx1-PQR por millón de usuarios Claro, Pqrx2-PQR por millón de usuarios Movistar, Pqrx3-PQR por millón de usuarios Tigo, Pqrx4-PQR por millón de usuarios Uff, Pqrt1-PQR total por año Claro, Pqrt2-PQR total por año Movistar, Pqrt3-PQR total por año Tigo, Pqrt4PQR total por año Uff.

La segunda fase correspondió a la formulación de los modelos que explican las relaciones entre el NSU y las variables independientes, para ello se usó el análisis de correlación estadística de Pearson y los métodos de regresión lineal. El objetivo del análisis de correlación fue seleccionar aquellas variables independientes susceptibles de explicar el indicador del NSU. La regresión lineal permitió establecer las ecuaciones matemáticas que explican y pronostican el NSU en función de las variables de interés. 
K. M. Prada-Ávila et al. / Efecto de las políticas de comercio y regulación en el nivel de satisfacción de los usuarios de telefonía móvil en Colombia

\section{RESULTADOS Y DISCUSIÓN}

El análisis de correlación de Pearson entre el NSU y las demás variables indicó una gran cantidad de correlaciones superiores a $\mathrm{r}>0,9$, por lo tanto y para evitar la presencia de colinealidad entre las variables independientes, se aplicó el proceso de centrado de variables descrito por Kleinbaum et al. [10] y Belsley [11]; las etiquetas de las variables centradas terminan con la letra $\mathrm{C}$ mayúscula.

Los análisis de correlación y colinealidad demostraron que las únicas variables independientes que permiten explicar y pronosticar el valor del NSU son las cifras de importaciones declaradas por la DIAN (DIAN CIF US\$), la penetración en el mercado del operador Claro (PM_CLARO) y la cantidad de teléfonos móviles homologados por la CRCom (CRC Tel_hom).

Con base en lo anterior, se procedió a la formulación de los modelos estadísticos que permiten explicar y pronosticar la magnitud del NSU total y por operador usando los siguientes criterios de aceptación: $\mathrm{R}$ cuadrado corregido $>0,6$; Valor $\mathrm{P}<0,05$; Significancia (Sig.) de las variables inde- pendientes $<0,05$; Factor Inflación Varianza (FIV) < 10; Índice de Condición < 5. Los estadísticos que soportan la obtención y calidad de los coeficientes que componen los modelos se presentan en la Tabla 4.

\subsection{Modelo para el NSU de Colombia}

El primer modelo presentado en (3) explica el $66,8 \%$ del NSU de Colombia a partir del valor de las importaciones de teléfonos móviles registrados por la DIAN, por ejemplo, un aumento de 100 millones de dólares en las importaciones de equipos, produce un incremento de 1,8\% en el NSU.

Total NSU $=0,597+1,77 \times 10^{-10} *(\mathrm{C} 1)$

La representación gráfica de la tendencia del NSU toal de Colombia se presenta en la Fig. 1. Dado que la CRCom no registra resultados de medición del NSU posteriores al 2009, se puede hacer la estimación con base en (3), así por ejemplo, según la DIAN las importaciones de teléfonos móviles en el 2012 ascendieron a 615 millones de dólares, entonces el NSU estimado para dicho año sería del $70 \%$.

Tabla 4. Resumen de estadísticos para los modelos. Fuente: Autores

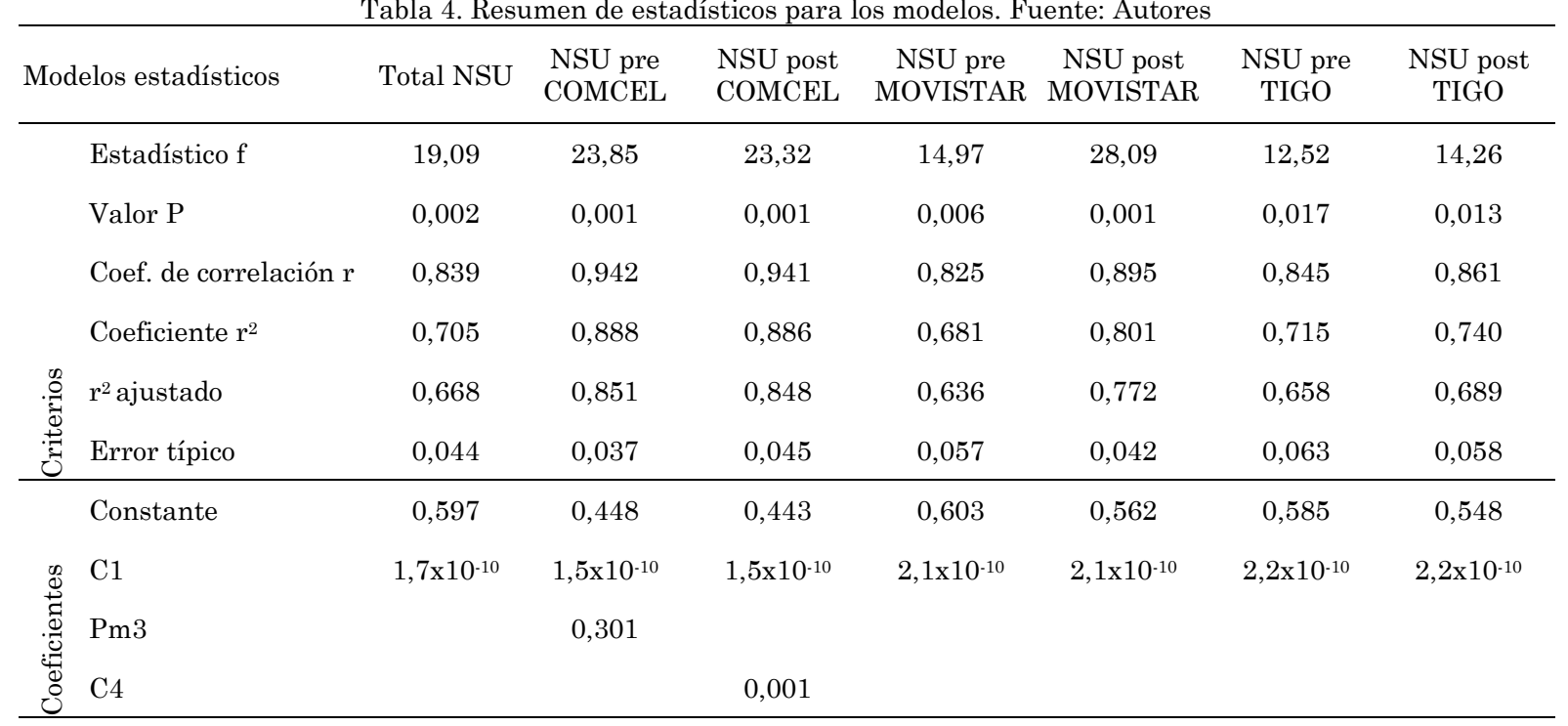


K. M. Prada-Ávila et al. / Efecto de las políticas de comercio y regulación en el nivel de satisfacción de los usuarios de telefonía móvil en Colombia

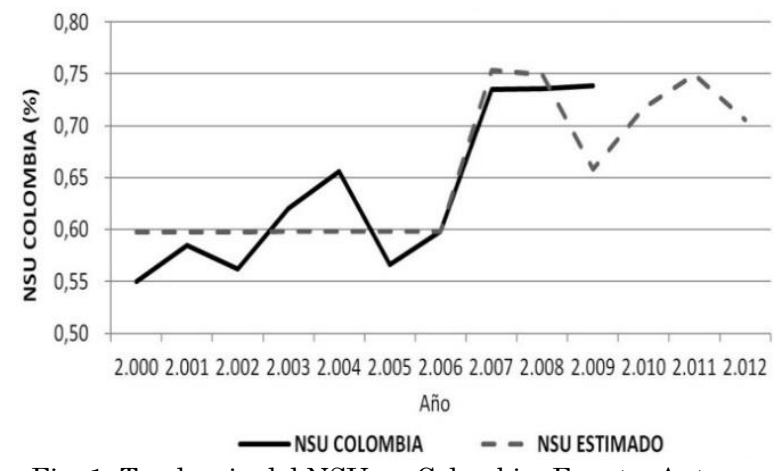

Fig. 1. Tendencia del NSU en Colombia. Fuente: Autores

\subsection{Modelo para el NSU de prepago Claro}

El segundo modelo presentado en (4) explica el $85,1 \%$ del NSU prepago de la empresa Claro a partir del valor de las importaciones de teléfonos móviles registrados por la DIAN y del porcentaje de penetración en el mercado de dicha compañía.

NSU pre Claro $=0 \begin{aligned} & 0,448+1,58 \times 10^{-10} * \mathrm{C} 1 \\ &+0,301 * \mathrm{Pm} 3\end{aligned}$

La representación gráfica de la tendencia del NSU de prepago Claro se presenta en la Fig. 2.

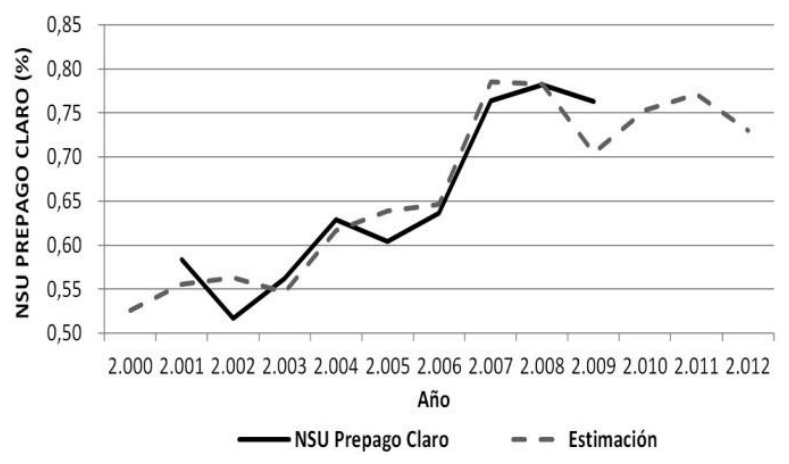

Fig. 2. Tendencia del NSU de prepago Claro. Fuente: Autores

El modelo indica que el NSU prepago de Claro puede aumentar por medio de un aumento de las cifras de importaciones de equipos móviles y mayor penetración en el mercado prepago, por ejemplo un aumento en 100 millones de dólares en las importaciones de equipos, producirá un incremento del NSU en 1,5\%. Por otro lado si Claro aumenta su participación en el mercado en $10 \%$ adicional, el NSU aumentará en 3,8\%, esto se puede explicar por los efectos de las tarifas más bajas entre usuarios del mismo operador, lo cual genera filiaciones masivas de grupos sociales.

\subsection{Modelo para el NSU de postpago Claro}

El tercer modelo presentado en (5) explica el $84,5 \%$ del NSU postpago de la empresa Claro a partir del valor de las importaciones de teléfonos móviles registrados por la DIAN y de las cifras de homologación de teléfonos móviles declarados por la CRCom.

$$
\begin{aligned}
\text { NSU pos Claro }= & 0.443+1,5 \times 10^{-10} * C 1 \\
& +1,46 \times 10^{-3} * C 4
\end{aligned}
$$

La representación gráfica de la tendencia del NSU de postpago Claro se presenta en la Fig. 3.

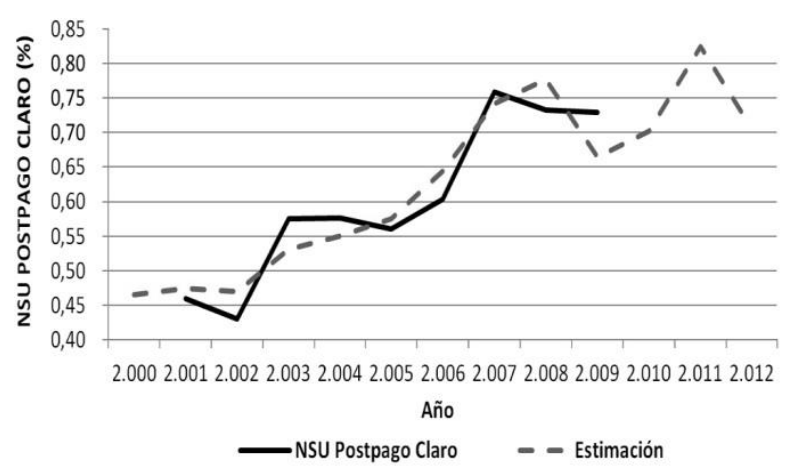

Fig. 3. Tendencia del NSU de postpago Claro. Fuente: Autores

En este modelo se mantiene la constante del efecto de las cifras de importaciones declaradas por la DIAN sobre el NSU postpago de la empresa Claro, sin embargo un efecto adicional y también incremental lo produce el número de referencias de teléfonos móviles homologados por la CRCom, el cual indica un aumento de 0,3\% en el NSU por cada 200 referencias homologadas en el año. 
K. M. Prada-Ávila et al. / Efecto de las políticas de comercio y regulación en el nivel de satisfacción de los usuarios de telefonía móvil en Colombia

\subsection{Modelos para el NSU de prepago y postpago Movistar}

Los NSU prepago y postpago de la empresa Movistar se explican y se pueden predecir a partir del valor de las importaciones de teléfonos móviles registrados por la DIAN. El modelo presentado en (6) para el NSU prepago explica el $63,6 \%$ del total de información y su representación gráfica se presenta en la Fig. 4.

NSU pre Movistar $=0,603+2,08 \times 10^{-10} * \mathrm{C} 1$

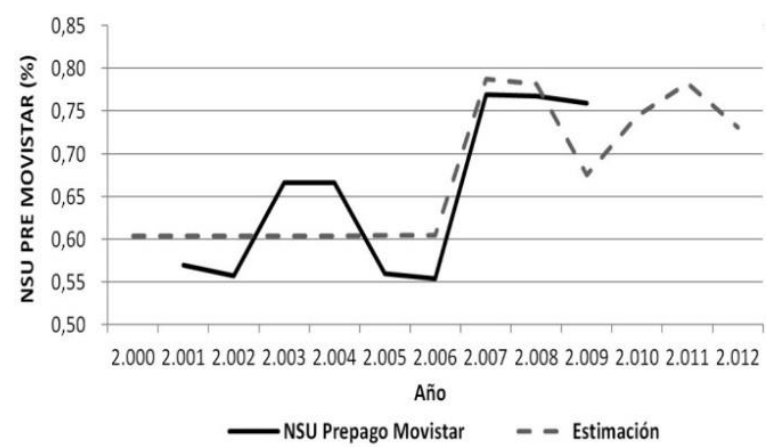

Fig. 4. Tendencia del NSU de prepago Movistar. Fuente: Autores

El modelo presentado en (7) para el NSU postpago explica el $77,2 \%$ y su representación gráfica se presenta en la Fig. 5.

NSU post Movistar

$$
=0.562+2.105 \times 10^{-10} * \mathrm{C} 1
$$

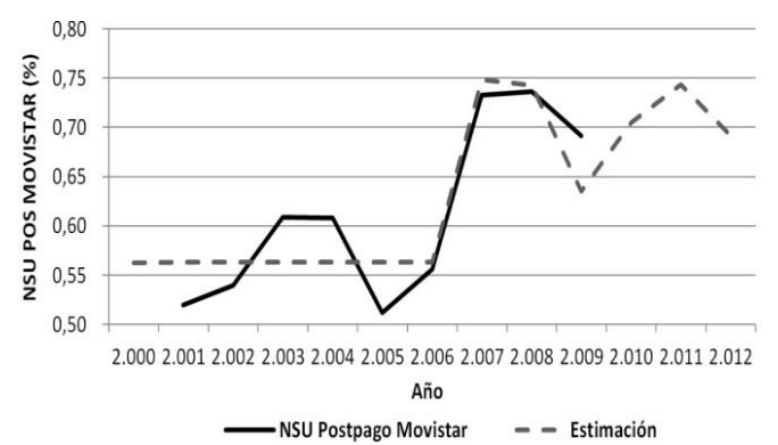

Fig. 5. Tendencia del NSU de postpago Movistar. Fuente: Autores

\subsection{Modelos para el NSU de prepago y postpago Tigo}

Los NSU prepago y postpago de la empresa Tigo se explican y se pueden predecir a partir del valor de las importaciones de teléfonos móviles registrados por la DIAN como se presenta en (8) y (9) respectivamente. El modelo para el NSU prepago explica el $65,8 \%$ y su representación gráfica se presenta en la Fig. 6 .

NSU pre Tigo $=0,585+2,24 \times 10^{-10} * \mathrm{C} 1$

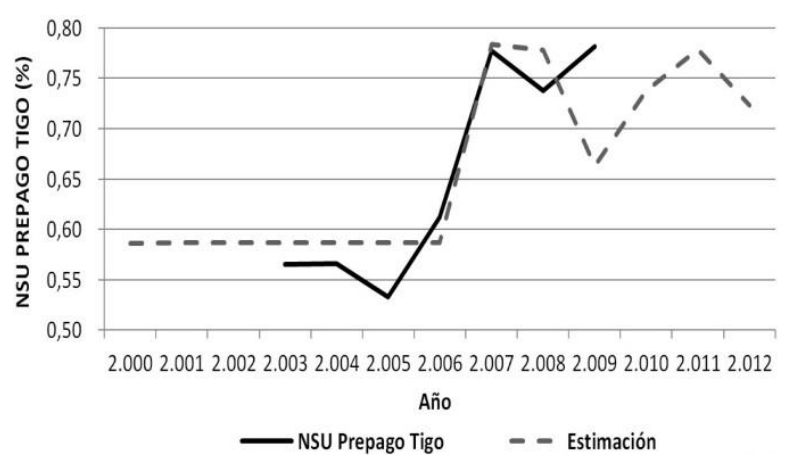

Fig. 6. Tendencia del NSU de prepago Tigo. Fuente: Autores

El modelo para el NSU postpago explica el $68,9 \%$ y su representación gráfica se presenta en la Fig. 7.

NSU post Tigo $=0.548+2,19 \times 10^{-10} * \mathrm{C} 1$

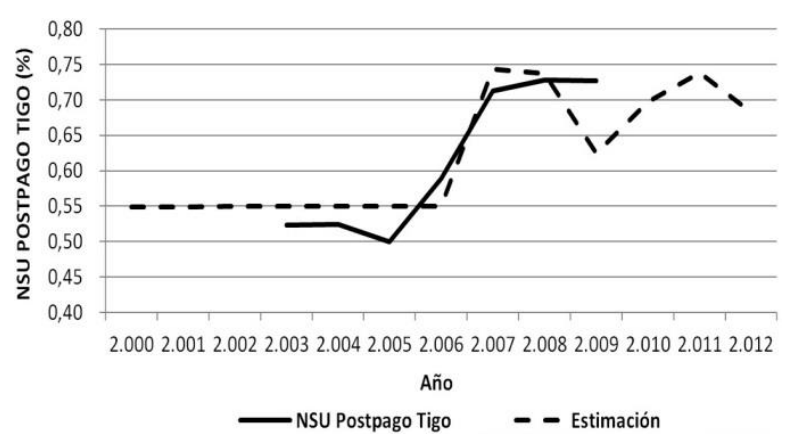

Fig. 7. Tendencia del NSU de postpago Tigo. Fuente: Autores

\section{CONCLUSIONES}

Se concluye que en el mercado de la telefonía móvil existen efectos regulatorios desconocidos para las instituciones del Estado, los cuales impiden fijar como prioridad la garantía de un nivel mínimo de calidad del servicio como requisito de operación dentro de éste, lo cual también es un 
K. M. Prada-Ávila et al. / Efecto de las políticas de comercio y regulación en el nivel de satisfacción de los usuarios de telefonía móvil en Colombia

reflejo de la demanda creciente que les garantiza a los operadores un aumento de ingresos más allá de la calidad del servicio ofrecida.

En esta investigación se encontró que las políticas legislativas, normativas y de importación de teléfonos móviles tienen un efecto sobre el Nivel de Satisfacción de los Usuarios - NSU del servicio de telefonía móvil en Colombia, explicado por la relación entre éste y las cifras de importaciones de terminales móviles, la cantidad de teléfonos homologados en el país y la distribución del mercado entre operadores regulada por el Estado.

Dichos factores corresponden a un punto de vista complementario al que tiene la Comisión de Regulación de Comunicaciones - CRCom sobre la medición del NSU, ya que su método actual de medición se centra en los atributos de calidad del servicio que los operadores otorgan a los usuarios y desconoce sus propios efectos regulatorios y los de aquellas entidades del Estado relacionadas con este mercado.

Todos los modelos que explican el NSU se relacionan con las cifras de importación de teléfonos móviles que registra la DIAN, lo cual refleja los efectos económicos de la evolución del mercado, por la oferta de nuevos servicios y la participación de nuevos operadores, sobre la satisfacción de los usuarios.

El NSU en Colombia se ha mantenido constante desde el 2007, a pesar de que la demanda aumentó en cerca de 9 millones de líneas hasta su último reporte en 2009; todas las estimaciones y pronósticos del NSU presentados en este artículo indican que este comportamiento no mejoraría en las mediciones al 2012, año en el que el número líneas fue superior en 14 millones al registrado en 2007. Esto indica que la satisfacción de los usuarios de telefonía móvil no es una prioridad para los operadores, toda vez que la demanda creciente les garantiza un aumento de ingresos a priori de la calidad del servicio ofrecida.

\section{REFERENCIAS}

[1] N. Nova and W. Pinzón, "Economic Rationality in Market Agents: Case Study of Mobile Telephone," in Industrial and Systems Engineering Research Conference, 2012.

[2] N. Nova, W. Pinzón, and R. Quintero, Hacia un nuevo modelo de cibernética, 1st ed. Bogotá: Universidad Distrital Francisco José de Caldas, 2013, p. 120.

[3] N. Nova-Arévalo, W. Pinzón-Rueda, and J. MezaÁlvarez, "Análisis de cuasi rentas generadas en el mercado de la telefonía móvil celular," Ing. Solidar., vol. 7, no. 12-13, pp. 27-34, 2011.

[4] Ministerio de Tecnologías de la Información y las Comunicaciones, "Sistema de Información Unificado del Sector de Telecomunicaciones - SIUST,” Bogotá, 2013.

[5] Comisión de Regulación de las Comunicaciones, "Condiciones de calidad en servicios de telecomunicaciones,” Bogotá, 2006.

[6] Comisión de Regulación de las Comunicaciones, Manual para la Medición del Nivel de Satisfacción del Usuario (NSU). Bogotá, 2007, p. 34.

[7] Comisión de Regulación de las Comunicaciones, "Información de Mercado - Medición indicador Nivel de Satisfacción al Usuario,” Bogotá, 2013.

[8] Centro Micro Datos, "Encuesta de satisfacción de usuarios de servicios de telecomunicaciones," Santiago de Chile, 2009.

[9] Ministerio de Industria Turismo y Comercio, "Estudio sobre la percepción de los usuarios acerca de la calidad de los principales servicios de telecomunicaciones," Madrid, 2009.

[10] D. G. Kleinbaum, L. L. Kupper, A. Nizam, and K. E. Muller, Applied Regression Analysis and Other Multivariables Methods, 4th ed. Cengage Learning, 1988, p. 928.

[11] D. Belsley, Conditioning Diagnostics: Collinearity and Weak Data in Regression. John Wiley \& Sons, 1991, p. 396. 